\title{
Schwannoma of the posterior branch of the great auricular nerve
}

\author{
Kwang Seog Kim, \\ Hyeok Lee, \\ Jun Ho Choi, \\ Jae Ha Hwang, \\ Sam Yong Lee
}

Department of Plastic and

Reconstructive Surgery, Chonnam

National University Medical School,

Gwangju, Korea

\begin{abstract}
Schwannoma, also known as neurilemmoma, is a tumor of the nerve sheath, which most often occurs in the peripheral nerves of the extremities. Schwannoma can be accompanied by symptoms such as pain, paresthesia, and Tinel sign; however, patients can also be asymptomatic. Here, we present the case of a 17-year-old woman who presented with a slowly growing, asymptomatic, postauricular mass that appeared 10 years prior. Ultrasonography was performed, and the mass was thought to be an epidermal inclusion cyst. However, the clinical manifestation during surgery was not correlated to an epidermal inclusion cyst, leading to the suspicion of schwannoma from the posterior branch of the great auricular nerve. After a meticulous dissection, schwannoma was diagnosed based on a permanent section biopsy. Postoperative complications and recurrence were not observed. Schwannoma in the peripheral nerve area of the face is rare. Therefore, an investigation of tumors that occur where the nerve passes using imaging and clinical features is necessary to confirm the diagnosis of schwannoma and to establish suitable treatment methods.
\end{abstract}

Keywords: Neurilemmoma / Peripheral nerves / Schwannoma

\section{INTRODUCTION}

Schwannoma, also known as neurilemmoma, is a rare, benign peripheral nerve sheath tumor originating from Schwann cells of the cranial, peripheral, and autonomic nerves. The vestibular nerve is most commonly involved when the cranial nerve is affected, but the facial nerve is occasionally involved as well. However, schwannomas rarely occur in the optic and olfactory nerves, which have thinner myelin sheaths. They also reportedly occur in the brachial plexus, cervical chain, and sympathetic trunk. Schwannomas usually grow slowly and present as a painless swelling over several years before other symptoms appear [1].

\section{Correspondence: Kwang Seog Kim}

Department of Plastic and Reconstructive Surgery, Chonnam National University

Medical School, 42 Jebong-ro, Dong-gu, Gwangju 61469, Korea

E-mail: pskim@chonnam.ac.kr

Received September 28, 2020 / Revised October 15, 2020 / Accepted November 16, 2020
Schwannoma rarely occurs in the peripheral nerves. Extracranial non-vestibular schwannomas are prevalent in the neck, particularly in the parapharyngeal space, while other common sites of occurrence include the supraclavicular neck (usually from the brachial plexus), oral cavity, and paranasal sinuses [2]. The most common nerves of origin for extracranial head and neck schwannomas are the vagus and sympathetic nerves. Schwannomas rarely occur in the subcutaneous layer of the head and neck. In addition, to the best to our knowledge, there has only been one reported case of schwannoma occurring in the great auricular nerve (GAN), which is a superficial branch of the cervical plexus that provides sensory innervation to the skin overlying the parotid gland, external ear, and postauricular region [3]. We report a rare case diagnosed as a schwannoma after excisional biopsy for a postauricular mass that was misdiagnosed as an epidermal inclusion cyst on ultrasonography (US). 


\section{CASE REPORT}

A 17-year-old woman presented with an asymptomatic, slow growing, postauricular mass, which appeared 10 years prior. The mass was palpable; however, pain, paresthesia, Tinel sign (shooting paresthesia in the distribution of the involved nerve), or other neurological deficits were not observed. Preoperative US revealed a hypoechoic mass $(1.5 \mathrm{~cm} \times 1.1 \mathrm{~cm} \times 0.5 \mathrm{~cm})$ in the subcutaneous layer, which was suspected to be an epidermal inclusion cyst (Fig. 1).

The mass was solid, round in shape, and softly fixed to the postauricular area (Fig. 2A). After dissecting the skin and soft tissue, a whitish yellow, shiny, solid mass was observed. Consid-

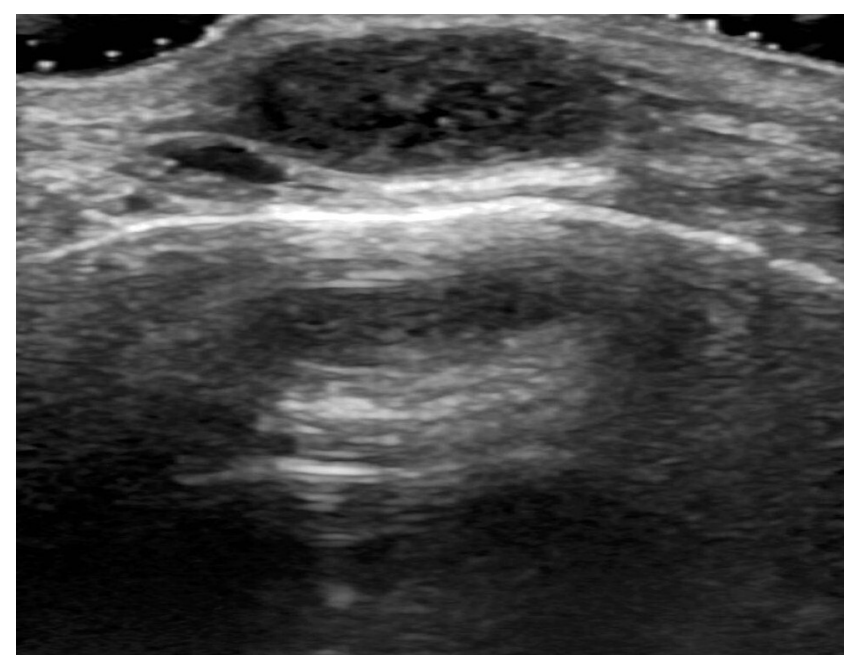

Fig. 1. Preoperative ultrasonography image. Ultrasonography reveals a hypoechoic mass in subcutaneous layer suspicious of epidermal inclusion cyst, measuring $1.5 \mathrm{~cm} \times 1.1 \mathrm{~cm} \times 0.5 \mathrm{~cm}$. ering the clinical features, we suspected a well-encapsulated schwannoma of the posterior branch of the GAN rather than an epidermal inclusion cyst. After confirming that fascicles were not involved through meticulous dissection, the tumor was completely shelled out during the excision (Fig. 2B). After confirming that the nerve was intact and there was no distortion of structures, the nerve and skin were closed in a layer-bylayer fashion (Fig. 2C). The surgical specimen was $1.7 \mathrm{~cm} \times 1.0$ $\mathrm{cm} \times 0.8 \mathrm{~cm}$ in size and was well-encapsulated (Fig. 3). Permanent section biopsy showed classic features of schwannoma, including Verocay bodies, and Antoni A and B areas (Fig. 4). Antoni A areas consist of compact spindle-shaped cells with twisted nuclei. The cells are arranged in short bundles with palisad-

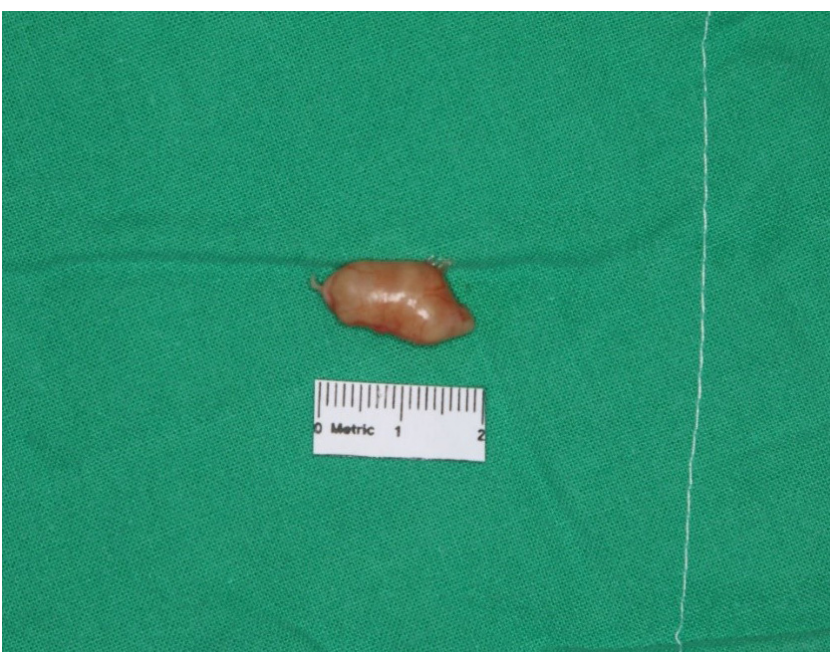

Fig. 3. Surgical specimen. The tumor appears white to yellow, shiny, solid, and well-encapsulated.
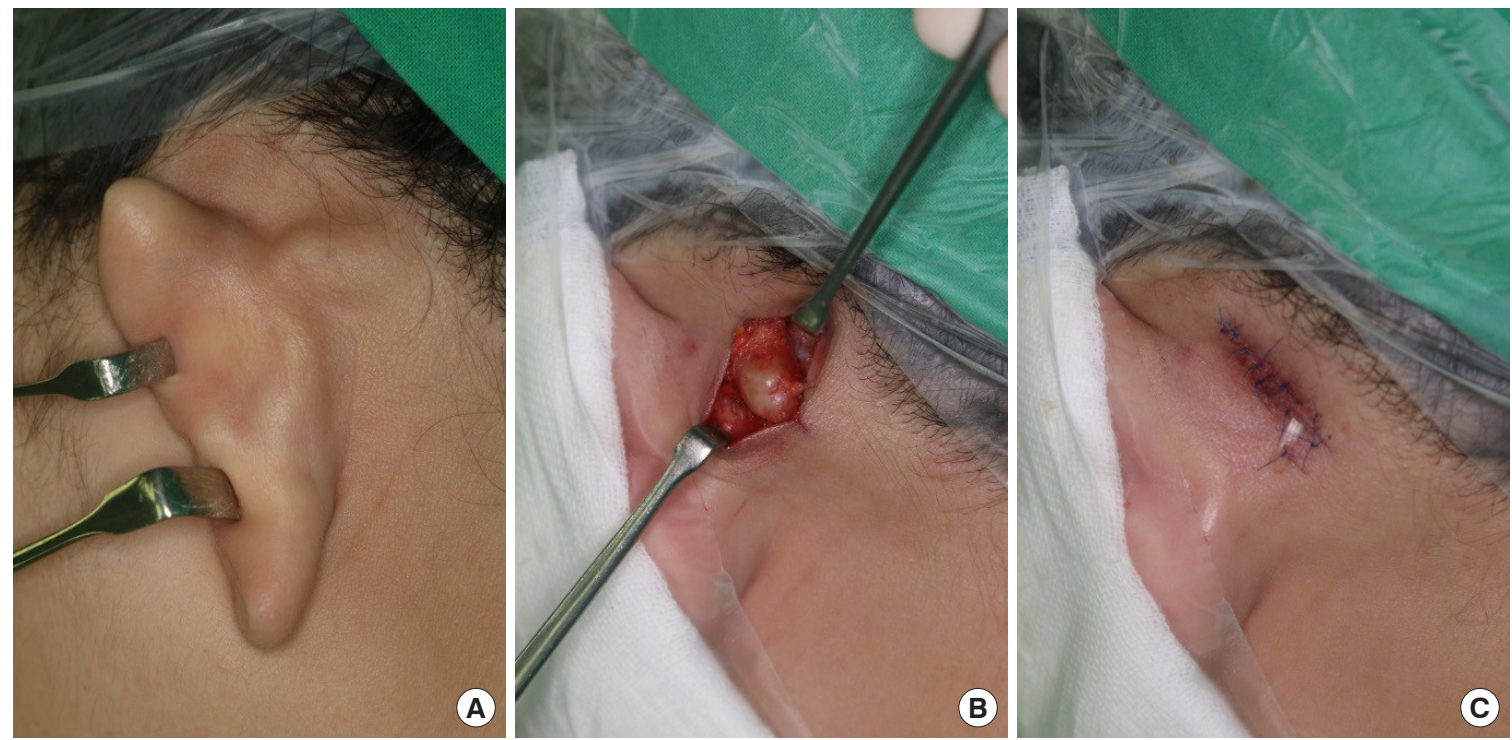

Fig. 2. Preoperative (A), intraoperative (B), and immediate postoperative (C) images. 

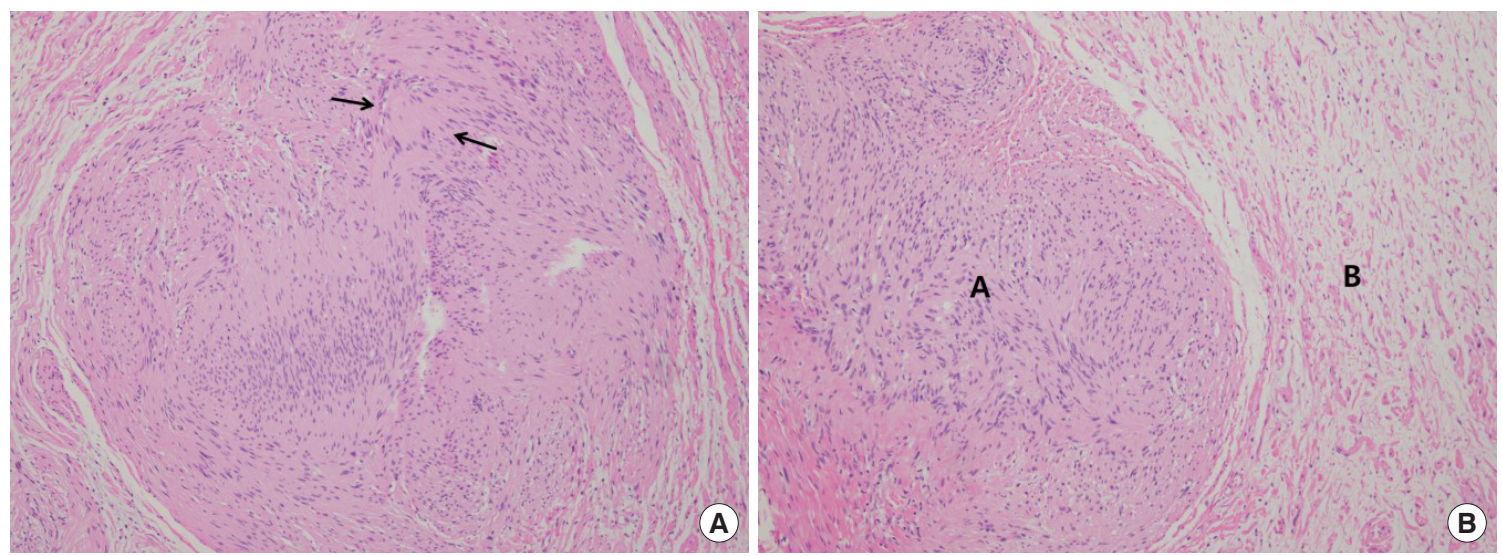

Fig. 4. Histologic images. (A) Verocay bodies; nuclear palisading about periphery of eosinophilic acellular cytoplasm (arrows). (B) Antoni A and $\mathrm{B}$ areas with compact spindle cells in A area and loose hypocellular tissue in $\mathrm{B}$ area $(\mathrm{H} \& \mathrm{E}, \times 100)$.

ing nuclei. Two dense rows of these well-aligned nuclei and cell processes are arranged elliptically to form a Verocay body. Antoni $\mathrm{B}$ areas display loosely arranged spindle cells within a myxoid matrix, which is susceptible to degeneration, hemorrhage, and cyst formation.

All stitches were removed 5 days after surgery, and there were no complications such as wound dehiscence, infection, and numbness. The patient showed no evidence of recurrence or neurological deficits upon follow-up for 3 years after complete excision.

\section{DISCUSSION}

Schwannoma is a benign peripheral nerve sheath tumor composed of proliferating Schwann cells. Schwannoma is relatively slow-growing and mostly benign. Less than $1 \%$ become malignant, degenerating into a form of cancer known as neurofibrosarcoma [4]. Schwannoma is usually asymptomatic, but as the size increases, symptoms and signs often appear due to the mass pressing on the adjacent anatomic structures [5]. A characteristic symptom of schwannoma is neurological deficit. Tumors originating in the sensory nerves may present as pain, those originating in the vagus nerve may present as hoarseness and globus sensations, and those originating in the facial nerves may present as facial palsy $[6,7]$. In this case, the schwannoma originated in the GAN. The GAN is a purely sensory nerve that arises from C2 and C3 spinal roots and fuses to the main trunk before penetrating the middle part of the sternocleidomastoid muscle (SCM). At the SCM, the GAN is in the most superficial position before bifurcating into anterior and posterior branches. The posterior branch travels on the surface of the SCM before reaching the mastoid area and ends at the postauricular area, which is responsible for sensation on the posteroinferior surface of the auricle [8]. Because the GAN is a purely sensory nerve, any pain, tenderness, neurological deficit, or Tinel sign in this area aids the diagnosis. However, in this case, there were no specific signs or symptoms as such. Therefore, these symptoms are only for reference in diagnosis and can be considered nonspecific.

The diagnosis of schwannoma is established by the pathological examination of tumor tissue. Histologically, the characteristic feature of schwannoma is the pattern of Antoni A and Antoni $\mathrm{B}$ areas, and Verocay bodies, as in this case. Positive staining of S-100 protein is specific to Schwann cells and is one of the histological criteria for diagnosis of schwannoma [9]. However, because other histological criteria were typical in this case, S-100 protein staining was not performed separately.

Complete surgical excision is the treatment of choice for schwannoma. When a schwannoma is limited to the nerve sheath and forms a capsule, it is rare that neurological deficit occurs after excision. In these cases, a schwannoma can be removed by including only part of the fascicles or by cutting the capsule. However, if there is significant fascicular involvement, neurorrhaphy or a nerve graft may inevitably be required to minimize neurologic deficit after en bloc excision [10,11]. Although schwannomas are benign, incomplete excision can cause a slow local recurrence over months to years [9].

In this case, the tumor occurred in a rare location, and there were no specific symptoms. The case also showed a similar pattern to an epidermal inclusion cyst on US; therefore, a schwannoma was not suspected. Thus, for tumors that occur where the nerve passes, a schwannoma must be differentiated using imaging such as computed tomography or magnetic resonance imaging before surgery, and a sufficient explanation should be provided to the patient regarding the diagnosis and treatment. 


\section{NOTES}

\section{Conflict of interest}

KSK, the editor-in-chief of the Archives of Craniofacial Surgery, is the corresponding author of this article. However, he played no role whatsoever in the editorial evaluation of this article or the decision to publish it. Except for that, no potential conflict of interest relevant to this article was reported.

\section{Ethical approval}

The study was approved by the Institutional Review Board of Chonnam National University Hospital (IRB No. CNUH-2020237) and performed in accordance with the principles of the Declaration of Helsinki. Written informed consent was obtained.

\section{Patient consent}

The patient provided written informed consent for the publication and the use of her images.

\section{ORCID}

\section{Kwang Seog Kim}

https://orcid.org/0000-0002-6766-4640

Hyeok Lee

Jun Ho Choi

Jae Ha Hwang

Sam Yong Lee https://orcid.org/0000-0003-4865-9595 https://orcid.org/0000-0002-4848-517X https://orcid.org/0000-0001-6992-8067 https://orcid.org/0000-0002-3185-2519

\section{REFERENCES}

1. Ozdemir O, Ozsoy MH, Kurt C, Coskunol E, Calli I. Schwannomas of the hand and wrist: long-term results and review of the literature. J Orthop Surg (Hong Kong) 2005;13:267-72.

2. Curioni OA, de Souza RP, Mercante AM, de Jesus AC, Pavelegeni A, Dedivitis RA, et al. Extracranial neurogenic tumors of the head and neck. Braz J Otorhinolaryngol 2015;81:604-9.

3. Ginsberg LE, Eicher SA. Great auricular nerve: anatomy and imaging in a case of perineural tumor spread. AJNR Am J Neuroradiol 2000;21:568-71.

4. Biswas D, Marnane CN, Mal R, Baldwin D. Extracranial head and neck schwannomas: a 10-year review. Auris Nasus Larynx 2007;34:353-9.

5. Kim KS, Ji SR, Kim HM, Kwon YJ, Hwang JH, Lee SY. Intercostal nerve schwannoma encountered during a rib-latissimus dorsi osteomyocutaneous flap operation. Arch Plast Surg 2015; 42:800-2.

6. Seo BF, Choi HJ, Seo KJ, Jung SN. Intraparotid facial nerve schwannomas. Arch Craniofac Surg 2019;20:71-4.

7. Choi JS, Park SM, Choi KU. A rare case of isolated schwannoma in infraorbital nerve. Arch Craniofac Surg 2018;19:231-4.

8. Lefkowitz T, Hazani R, Chowdhry S, Elston J, Yaremchuk MJ, Wilhelmi BJ. Anatomical landmarks to avoid injury to the great auricular nerve during rhytidectomy. Aesthet Surg J 2013; 33:19-23.

9. Kikuta S, Jenkins S, Kusukawa J, Iwanaga J, Loukas M, Tubbs RS. Ansa cervicalis: a comprehensive review of its anatomy, variations, pathology, and surgical applications. Anat Cell Biol 2019;52:221-5.

10. Tang CY, Fung B, Fok M, Zhu J. Schwannoma in the upper limbs. Biomed Res Int 2013;2013:167196.

11. Hwang JH, Lee DG, Sim HS, Kim KS, Lee SY. Intramasseteric schwannoma treated with facelift incision and retrograde facial nerve dissection. Arch Craniofac Surg 2019;20:388-91. 